\title{
A global optimisation study of the low-lying isomers of the alumina octomer $\left(\mathrm{Al}_{2} \mathrm{O}_{3}\right)_{8}$
}

\author{
David Gobrecht ${ }^{\text {齐a }}$, Leen Decin ${ }^{\mathrm{a}}$, Sergio Cristallo ${ }^{\mathrm{b}, \mathrm{c}}$, Stefan T. Bromley ${ }^{\mathrm{d}, \mathrm{e}}$ \\ ${ }^{a}$ Instituut voor Sterrenkunde, KU Leuven, Celestijnenlaan 200D, B-3001 Leuven, Belgium \\ ${ }^{b}$ INAF - Osservatorio Astronomico d'Abruzzo, Via mentore maggini s.n.c., I-64100 Teramo, Italy \\ ${ }^{c}$ INFN - Sezione di Perugia, via A. Pascoli, I-06123, Perugia, Italy \\ ${ }^{d}$ Departament de Ciència de Materials i Química Física $\mathbb{E}$ Institut de Química Teórica $i$ \\ Computacional (IQTCUB), Universitat de Barcelona, E-08028 Barcelona, Spain \\ ${ }^{e}$ Institució Catalana de Recerca i Estudis Avançats (ICREA), E-08010 Barcelona, Spain
}

\begin{abstract}
We employ the Monte-Carlo Basin-Hopping (MC-BH) global optimisation technique with inter- atomic pair potentials to generate low-energy candidates of stoichiometric alumina octomers $\left(\left(\mathrm{Al}_{2} \mathrm{O}_{3}\right)_{8}\right)$. The candidate structures are subsequently refined with density functional theory calculations employing hybrid functionals (B3LYP and PBE0) and a large basis set $(6-311+\mathrm{G}(\mathrm{d}))$ including a vibrational analysis. We report the discovery of a set of energetically low-lying alumina octomer clusters, including a new global minimum candidate, with shapes that are elongated rather than spherical. We find a stability limit for these and smaller-sized clusters at a temperature of $T \simeq 1300-1450 \mathrm{~K}$ corresponding to a phase transition in liquid alumina.
\end{abstract}

Keywords: aluminum oxide, molecular clusters, global optimisation, nucleation

\section{Introduction}

Alumina clusters play a role in atmospheric chemistry [1. Being artificially produced by rocket flights, $\mathrm{Al}_{2} \mathrm{O}_{3}$ cluster aerosols impact the Earth's atmospheric chemistry as they act as catalysts. Moreover, owing to their high thermal stabilities and (near)-infrared properties, alumina clusters are promising candidates to form the seed nuclei of dust formation in oxygen-rich AGB stars [2, 3, 4, 5. Although silicate dust constitutes the major part of oxygen-rich cosmic dust, its nucleation solely from gas-phase precursors is energetically hampered and explicitely ruled out for $\mathrm{SiO}[6,7$, and $\mathrm{MgO}$ [8, 9]. Instead, it is more likely that the silicate dust forms on top of pre-existing seed nuclei. These seed nuclei must form from available atoms and molecules, and have to sustain the extreme thermodynamic conditions close to the stellar surface. In oxygen- dominated regimes, the latter requirement are fulfilled by highly refractory metal oxides such as alumina $\left(\mathrm{Al}_{2} \mathrm{O}_{3}\right)$ and titania $\left(\mathrm{TiO}_{2}\right)$. Studies on stardust grains from pristine meteorites show

\footnotetext{
corresponding author

Email address: david.gobrecht@kuleuven.be (David Gobrecht ${ }^{\text {th }}$ )

Preprint submitted to Chemical Physics Letters
}

October 5, 2018 
larger sizes and greater amounts of alumina, compared with titania [10, 11]. Thus, we presume that it is most likely that alumina initiates stardust synthesis in oxygen-rich conditions.

Apart from a pure scientific interest alumina clusters are also of practical use. Owing to their large refractories, high hardness, high stabilities, high insulation capabilities, and optical transparency [12] alumina clusters serve as components for diverse purposes and applications. Over the past few decades, nano-sized alumina fibers have been synthesized by diverse techniques (hydrothermal or solvothermal, sol-gel techniques, electrospinning, extrusion, chemical vapor deposition) [13] and have attracted a lot of attention. The fibers are used in electronics, informatics, and communications [14. Moreover, owing to its bio-compatibility, alumina nanofibers are also used for drug delivery. In the laboratory, clusters of nano-sized alumina can be produced in deionized water by laser ablation of aluminum targets [15.

There are several bulk polymorphs for alumina. The most stable polymorph at standard conditions ( $\mathrm{p}=1 \mathrm{~atm}, \mathrm{~T}=298 \mathrm{~K}$ ) is the hexagonally, closed-packed $\alpha$-alumina which is the main component of corundum [16]. Other alumina polymorphs are $\beta, \gamma$, $\delta, \eta$ and $\chi$-alumina that represent the most stable alumina bulk forms at elevated temperatures. All these crystalline bulk forms have trivalent $\mathrm{Al}^{3+}$ cations and divalent $\mathrm{O}^{2-}$ in common. In this work, we compare the properties of our individual clusters with respect to each other, and with $\alpha$ alumina. Previous studies have shown that alumina clusters substantially deviate from the bulk-like analogs [17, 18. In the size regime with dimension $\mathrm{d} \leq 1 \mathrm{~nm}$ (which corresponds to the size range from the monomer to the octomer, $\mathrm{n}=8$ ), diverse geometries (flat, cage-like, compact) represent the most stable isomer structures [19, 20, 21]. The alumina octomer corresponds to the first cluster size at which we find differences with previously reported candidate global minima in the literature. We revisit also smaller cluster sizes $(n<8)$ and we find the same structural isomers as found in the thorough study by 20. As a result of strong (attractive and repulsive) Coulomb forces between $\mathrm{O}$-anions and $\mathrm{Al}$ - cations, all favourable clusters have a strictly alternating cation-anion ordering in common.

This letter is organized as follows. In Section 2, we describe the methods to find lowenergy alumina octomer structures and their subsequent refinement at the DFT (Density Functional Theory) level. The results of our calculations are presented in Section 3. Finally, we discuss and summarize our new findings in Section 4.

\section{Methods}

\subsection{Global optimisation searches}

An alumina octomer, i.e. $\left(\mathrm{Al}_{2} \mathrm{O}_{3}\right)_{8}$, has 40 atoms and a total of $3 \times 40=120$ degrees of freedom resulting in a huge configurational space. A complete exploration of this space would require enormous amount of computational power and is beyond the current computing ability. To reduce the number of possible structural configurations to explore (and hence also the computational effort) a global optimisation search for low-energy octomer isomers is performed. We employ the Monte-Carlo Basin-Hopping (MC-BH) global optimisation technique 22] with inter-atomic pair potentials of an Al-O system to find candidate structures. For our purposes, we use an in-house, modified version of the GMIN programme 23. The general form of the inter-atomic Buckingham pair potential (including the Coulomb potential) reads: 
Table 1: The parameter ranges used in this study to compute the inter-atomic Buckingham pair potential. Charges $\mathrm{q}(\mathrm{Al})$ and $\mathrm{q}(\mathrm{O})$ are given in atomic units, $\mathrm{A}$ in $\mathrm{eV}, \mathrm{B}$ in $\AA \mathrm{C}$ in $\mathrm{eV} \AA^{-6}$.

\begin{tabular}{ll|lll|lll}
$\mathrm{q}(\mathrm{Al})$ & $\mathrm{q}(\mathrm{O})$ & $\mathrm{A}(\mathrm{Al}-\mathrm{O})$ & $\mathrm{B}(\mathrm{Al}-\mathrm{O})$ & $\mathrm{C}(\mathrm{Al}-\mathrm{O})$ & $\mathrm{A}(\mathrm{O}-\mathrm{O})$ & $\mathrm{B}(\mathrm{O}-\mathrm{O})$ & $\mathrm{C}(\mathrm{O}-\mathrm{O})$ \\
\hline & & & & & & & \\
$+3,+2.4$ & $-2,-1.6$ & 2409.5 & 0.2649 & 0.0 & 22764.0 & 0.14 & 27.88 \\
$+3,+2.4$ & $-2,-1.6$ & 4534.2 & 0.2649 & 0.0 & 25.410 & 0.6937 & 32.32
\end{tabular}

$$
U\left(r_{i j}\right)=\frac{q_{i} q_{j}}{r}+A \exp \left(-\frac{r_{i j}}{B}\right)-\frac{C}{r_{i j}^{6}}
$$

where $r_{i j}$ is the relative distance of two atoms, $q_{i}$ and $q_{j}$ the charges of atom $i$ and $j$, respectively, and $A, B$ and $C$ the Buckingham pair parameters. The first term in equation 1 represents the Coulomb law, the second term the short-range, steric repulsion term accounting for the Pauli principle, and the last term describes the attractive vander-Waals interaction. The potential describes the repulsion and attraction of charged particles, in this case, of aluminum and oxygen ions within a $\left(\mathrm{Al}_{2} \mathrm{O}_{3}\right)_{8}$ cluster. As lightest members of group III and group VI elements in the periodic table, $\mathrm{Al}$ and $\mathrm{O}$ form ionic bonds. The strong difference in electro-negativity, $\Delta \mathrm{EN}=2.03$, strongly suppresses the presence of covalent bonds. To reduce the probability to miss stable configurations in our searches we perform test calculations by swapping $\mathrm{Al}$ and $\mathrm{O}$ atoms of the most stable configurations. These tests account for atomic segregation (i.e. covalent bonds between identical atoms). The steric repulsion term is motivated by the fact that atoms are not dot-like but occupy a certain volume in space. We use the simplified form of the Buckingham pair potential and omit the repulsive $\mathrm{r}^{-12}$ Lennard-Jones term. Effectively, the latter term acts only on very short distances which is already taken into account by values of the parameters A and B. In the present approach we approximately account for the polarization effects by reducing the formal charges of the Al-cations and the O-anions by $10-20 \%$.

We apply two different parameter sets that are listed in Table 1. The first listed set (set 1) is commonly used for structure optimisation of Al-O systems (see e.g. 21]). The second parameter set in the last line of Table 1 corresponds to $\mathrm{Ag}^{+3}-\mathrm{O}^{-2}$ parameters published by 24. With exception of the value of $\mathrm{A}(\mathrm{Ag}-\mathrm{O})$, the other parameters are identical to the shell $\mathrm{Al}^{3+} \mathrm{O}^{2-}$ set of 25 . Set 2 complements set 1 in the sense that it covers a different parameter space accounting for structural families that could not (or hardly) be found with set 1 . For example, set 1 tends to result in compact geometries, but structural families like void cages and open-cage-like clusters are underrepresented. Both parameter sets are explored with a variety of different seed structures (i.e. initial geometries).

In summary, we use two different parameter sets, dozens of seed structures and different temperatures $(\mathrm{T}=300-3000 \mathrm{~K})$ in order to cover an extensive number of structural possibilities. Although the use of force fields is an approximation, their use enables us to perform tractable thorough searches. With our force-field approach we hope to have minimized the probability to miss a stable alumina octomer. 


\subsection{Optimisation at the DFT level}

Once multiple sets of candidate structures with different seed geometries, temperatures and parameter sets are found, we refine the 100 most favourable candidate structures with hybrid density functional theory (DFT) methods. We use two different density functionals, B3LYP [26] and PBE0 [27], in combination with the $6-311+\mathrm{G}(\mathrm{d})$ basis set and perform the calculations with the help of the computational chemistry software package Gaussian09 [28. The DFT calculations are performed at $0 \mathrm{~K}$ and 0 atm. In the Born-Oppenheimer approximation used here the Potential Energy Surface (PES) does not depend on temperature. Hence, the optimised cluster geometry is also temperature-independent. However, the vibrational population and the computation of the thermodynamic quantities (enthalpy, entropy, Gibbs free energy) depend on temperature. The thermal corrections are evaluated at standard conditions for temperature and pressure $(\mathrm{T}=298.15 \mathrm{~K}, \mathrm{p}=1 \mathrm{~atm})$. We include a vibrational analysis to calculate appropriate partition functions for any other conditions and to exclude possible transition states.

\section{Results}

\subsection{Minima structures}

Our lowest energy candidate global minimum octomer $\left(\mathrm{Al}_{2} \mathrm{O}_{3}\right)_{8}$ structure (hereafter 8A) calculated at the B3LYP $/ 6-311+\mathrm{G}(\mathrm{d})$ level of theory is displayed in Figure 1. The structure has a rotational symmetry and belongs to the $\mathrm{C}_{2}$ point symmetry group. Its potential energy is lower by $0.15 \mathrm{eV}$ relative to the next higher-lying isomer. With the PBE0/6-311+G(d,p) functional/basis set $8 \mathrm{~A}$ represents the second lowest-energy structure (relative energy difference of $0.17 \mathrm{eV}$ with respect to $8 \mathrm{~B}$ - see below) that we find. The HOMO-LUMO gap in the B3LYP (PBE0)- optimised new structure is 4.93 $(5.45) \mathrm{eV}$.
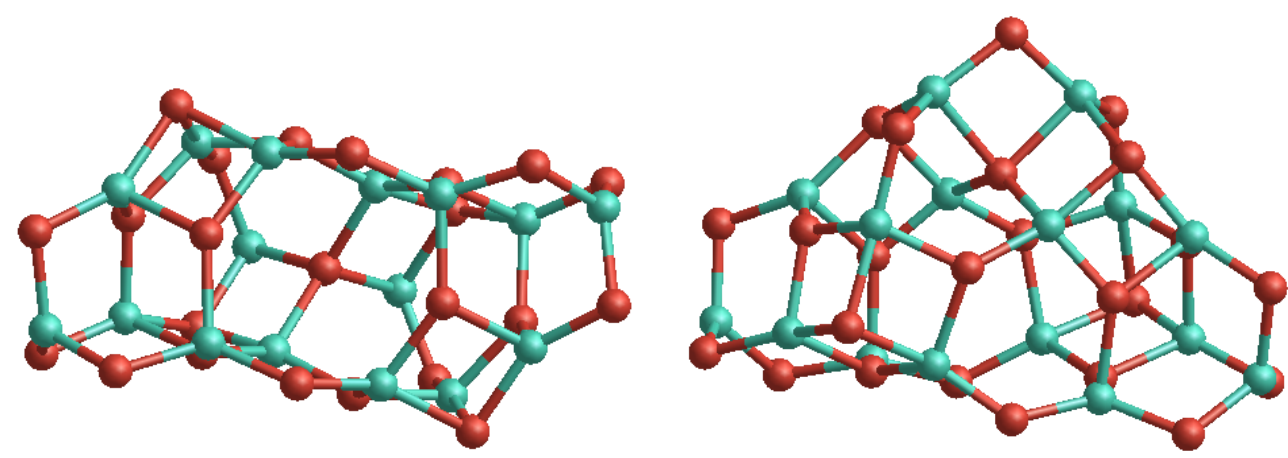

Figure 1: The global minimum structure $\mathbf{8 A}$ at the B3LYP/6-311+G(d) level seen from two distinct perspectives (that are perpendicular to each other). Al atoms are in green, $\mathrm{O}$ atoms in red.

Our search also resulted in the discovery of another alumina octomer cluster (hereafter $\mathbf{8 B}$ ) that is displayed in Figure 2, 8B shows a $\mathrm{C}_{s}$-symmetric structure. However, during the optimisation at the DFT level, the symmetry of $8 \mathrm{~B}$ is distorted and the assigned point symmetry group for $8 \mathrm{~B}$ is $\mathrm{C}_{1}$. Structure $8 \mathrm{~B}$ is the lowest-energy configuration 

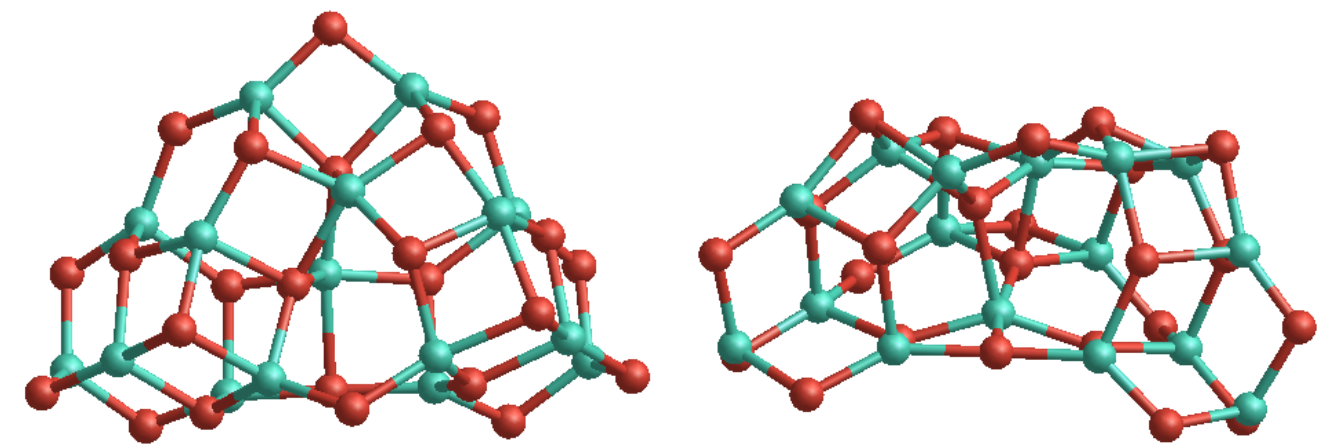

Figure 2: The global minimum structure $\mathbf{8 B}$ at the $\mathrm{PBE} 0 / 6-311+\mathrm{G}(\mathrm{d})$ level seen from two distinct perspectives

that we find with the PBE0 functional and the second-lowest (relative energy $0.15 \mathrm{eV}$ ) with the B3LYP functional. The HOMO-LUMO gap in the B3LYP (PBE0)-optimized structure $8 \mathrm{~B}$ is 4.67 (3.60) eV which is less than for $8 \mathrm{~A}$.

Further energetically low-lying alumina octomer isomers that have been found in this study are shown in Figure 3 All of the latter local minima structures are not symmetric and belong to the $\mathrm{C}_{1}$ point group. Structure $8 \mathrm{C}$ has relative potential energy $0.35 \mathrm{eV}$ $(0.25 \mathrm{eV})$ above the minimum with the B3LYP (PBE0) functional.

$8 \mathrm{D}, 8 \mathrm{E}$ and $8 \mathrm{~F}$ are located $0.97(1.14), 0.98(0.71)$ and $1.43(1.24) \mathrm{eV}$ above the global minimum structure $8 \mathrm{~A}(8 \mathrm{~B})$, respectively. In particular, we note that $8 \mathrm{D}$ and $8 \mathrm{E}$ are almost degenerate at the B3LYP level of theory, but have a significant difference $(0.43$ $\mathrm{eV}$ ) as calculated with the PBE0 functional. All structures - except $8 \mathrm{~F}$, but including $8 \mathrm{~A}$ and $8 \mathrm{~B}$ - exhibit an overall elongated geometry where one dimension $(\sim 9-10 \AA)$ is significantly longer than the other two dimensions (typically $\sim 6-7 \AA$ ). This may suggest an inherent tendency for a deviation from sphericity in a homogeneous nucleation scenario for alumina.

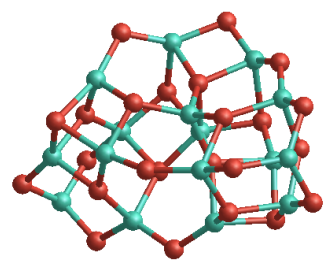

8C $0.35(0.25) \mathrm{eV}$

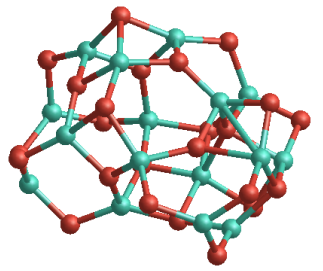

8D 0.97 (1.14) eV

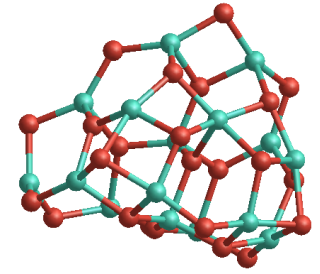

8E $0.98(0.71)$ eV

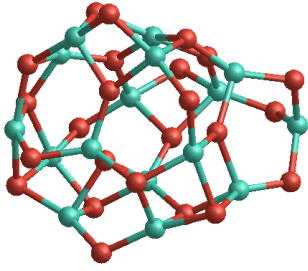

8F $1.43(1.24) \mathrm{eV}$

Figure 3: Energetically low-lying alumina octomer structures with energetic ordering according to DFT optimisations using the B3LYP functional.

In Figure 4, the predicted global minimum structure in ref. [19. (hereafter $\mathbf{8 G}$ ) is shown. $8 \mathrm{G}$ has two mirror planes, two rotational symmetries and belongs to the $\mathrm{D}_{2 d}$ space group. $8 \mathrm{G}$ has a potential energy $1.56(0.88) \mathrm{eV}$ above the minima structures $8 \mathrm{~A}$ (8B). 

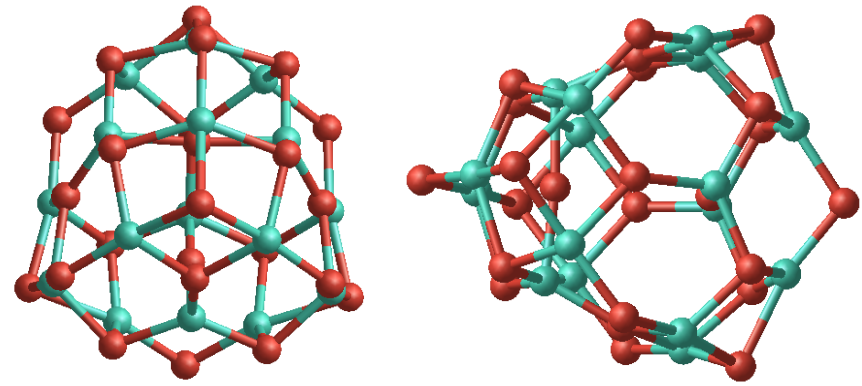

Figure 4: The minimum structure $\mathbf{8 G}$ seen from two distinct perspectives.

Five other low-lying alumina octomer isomers were predicted in ref. 21, see Figure 7 in their paper) using a gradient-based genetic algorithm (GA-LBFGS) and a Buckingham pair potential. We have also found the latter five isomers and investigated them on the DFT level of theory. Structures A, B, D and E in ref. 21] relax to structure 8G during a DFT optimisation with the hybrid functionals B3LYP and PBE0 and thus do not represent truly distinct structural isomers. The investigation of structures $\mathrm{C}$ and $\mathrm{E}$ in Figure 7 in ref. [21] at a DFT level results in the same isomer (see Figure 5) with a relative energy $2.77(1.89) \mathrm{eV}$ above $8 \mathrm{~A}(8 \mathrm{~B})$. It has a mirror plane and space group $\mathrm{C}_{2 v}$.
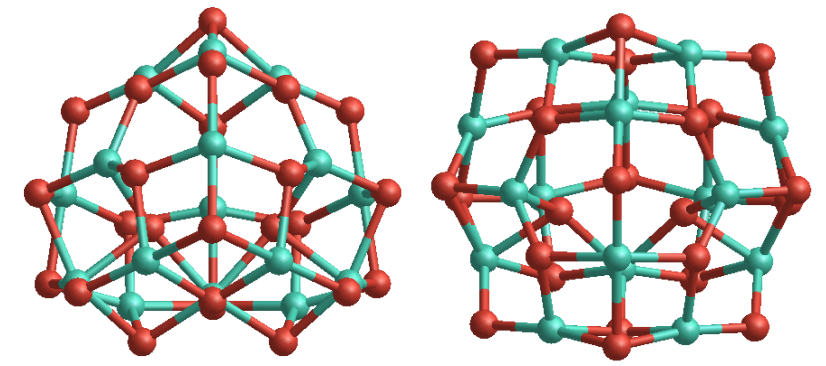

Figure 5: The stable structure $\mathbf{8 H}$ seen from two distinct perspectives.

Recently, the structural family of $\left(\mathrm{Al}_{2} \mathrm{O}_{3}\right)_{n}$ hollow spheres and bubble clusters has been studied for certain sizes $(n=10,12,16,18,24$ and 33) 29]. The latter structures are characterized by Al-atoms with coordination number 3 and O-atoms that are 2- coordinated. For $n=8$, we also find a member of the hollow spheres, or "bubble" family (see Figure 6. Cluster 8I has a very symmetric peanut-shaped geometry and belongs to the $\mathrm{D}_{2 h}$ point group. It has an electronic energy 5.31 (7.67) eV above the global minimum. We report the finding of six new isomer structures $(8 \mathrm{~A}, 8 \mathrm{~B}, 8 \mathrm{C}, 8 \mathrm{D}, 8 \mathrm{E}, 8 \mathrm{~F})$. The previously reported structures $8 \mathrm{G}, 8 \mathrm{H}$ and $8 \mathrm{I}$ have potential energies that are significantly higher than $8 \mathrm{~A}-8 \mathrm{~F}$.

\subsection{Rotational constants}

The rotational constants of the two isomers $8 \mathrm{~A}$ and $8 \mathrm{~B}$ are tabulated in Table 2 . The rotational constants obtained with the PBE0 functional are marginally larger $(\sim 1 \%)$ 


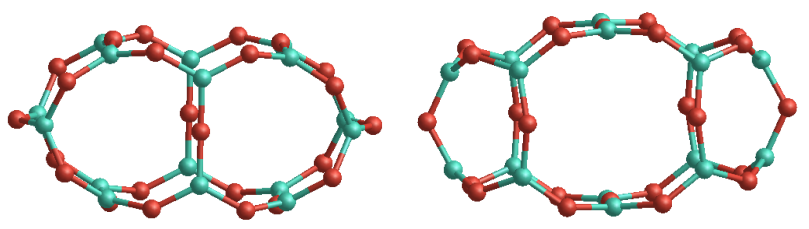

Figure 6: The stable structure 8I

Table 2: Rotational constants for $8 \mathrm{~A}$ and $8 \mathrm{~B}$ in $(\mathrm{GHz})$

\begin{tabular}{l|rr|rr}
$\begin{array}{l}\text { Rot. Constants } \\
\text { GHz }\end{array}$ & 8A & \multicolumn{3}{|c}{8 B } \\
(B3LYP) & PBE0 & B3LYP & PBE0 \\
\hline $\mathrm{B}_{x}$ & 0.113953 & 0.115163 & 0.090181 & 0.091319 \\
$\mathrm{~B}_{y}$ & 0.062404 & 0.063075 & 0.084215 & 0.085045 \\
$\mathrm{~B}_{z}$ & 0.056707 & 0.057285 & 0.084212 & 0.085042
\end{tabular}

that those obtained with the B3LYP functional. This is a consequence of the slightly more compact geometry of the PBE0 optimized structures leading to lower moments of inertia and higher rotational constants. Owing to its $\mathrm{C}_{2}$ symmetry $8 \mathrm{~B}$ has two almost identical constants $\left(\mathrm{B}_{y}\right.$ and $\left.\mathrm{B}_{z}\right)$.

\subsection{Bond distances, coordination and charges}

The Al-O bond distances of the clusters $8 \mathrm{~A}, 8 \mathrm{~B}$, and $8 \mathrm{G}$, and of the crystalline bulk ( $\alpha$-alumina) are shown in Figure 7. Al-Al and O-O bonds do not appear owing to the strong Coulomb repulsion of ions with the same (or similar) charge. The Al-O bond lengths of the clusters $8 \mathrm{~A}, 8 \mathrm{~B}$, and $8 \mathrm{G}$, as well as of $\alpha$-alumina form two populations, at short and at long bond lengths. The short bond peaks at $1.74 \AA$, and the long bond at 1.845 for structure $8 \mathrm{~A}$, for $8 \mathrm{~B}$ the peaks are located at $1.745 \AA$ and $1.855 \AA$, whereas they lie around $1.765 \AA$ and $1.850 \AA$ for $8 \mathrm{G}$. Also in the bulk phase, $\alpha$ alumina exhibits Al-O bonds with two different lengths being located at $1.972 \AA$ and at $1.855 \AA$.

It is apparent that the clusters and the bulk exhibit bond lengths of around $1.85 \AA$. However, the most prominent feature of $\alpha$-alumina located at $1.972 \AA$ is largely absent in the clusters and vice versa, inter-atomic distances smaller than $1.8 \AA$ do no appear in $\alpha$-alumina, but account for a significant fraction of the cluster bonds. In $8 \mathrm{~A}, 14$ out of $16 \mathrm{Al}$ cations are 4-coordinated, the remaining $2 \mathrm{Al}$ are 3-coordinated, whereas 11 oxygen anions are 2-coordinated, $12 \mathrm{O}$ atoms 3-coordinated and just one 4- coordinated. We count $62 \mathrm{Al}-\mathrm{O}$ bonds in total. The situation is similar for isomer 8B: It has $124-$ coordinated, 2 3-coordinated and 25 - coordinated Al cations, respectively. The oxygen anions are 2-coordinated (10), 3- coordinated (12) and 4-coordinated (2). A total of $64 \mathrm{Al}-\mathrm{O}$ bonds is present in 8B. Contrary, structure 8G exhibits 12 4-coordinated, 4 5coordinated, but no 3 -coordinated $\mathrm{Al}$ cations. Also in the oxygen coordination $8 \mathrm{G}$ differs from $8 \mathrm{~A}$ and 8B: $16 \mathrm{O}$ anions are 3 coordinated, $6 \mathrm{O}$ ions are 2-coordinated and $2 \mathrm{O}$ ions are 4-coordinated, respectively. The number of Al-O bonds is 68 and thus slightly higher than in $8 \mathrm{~A}$ and $8 \mathrm{~B}$. In $\alpha$-alumina, $\mathrm{Al}$ cations are 6 -coordinated and $\mathrm{O}$ anion 4coordinated. The average coordination in the crystalline bulk is (as expected) higher than for the clusters. In the clusters, a considerable fraction of the atoms are located 


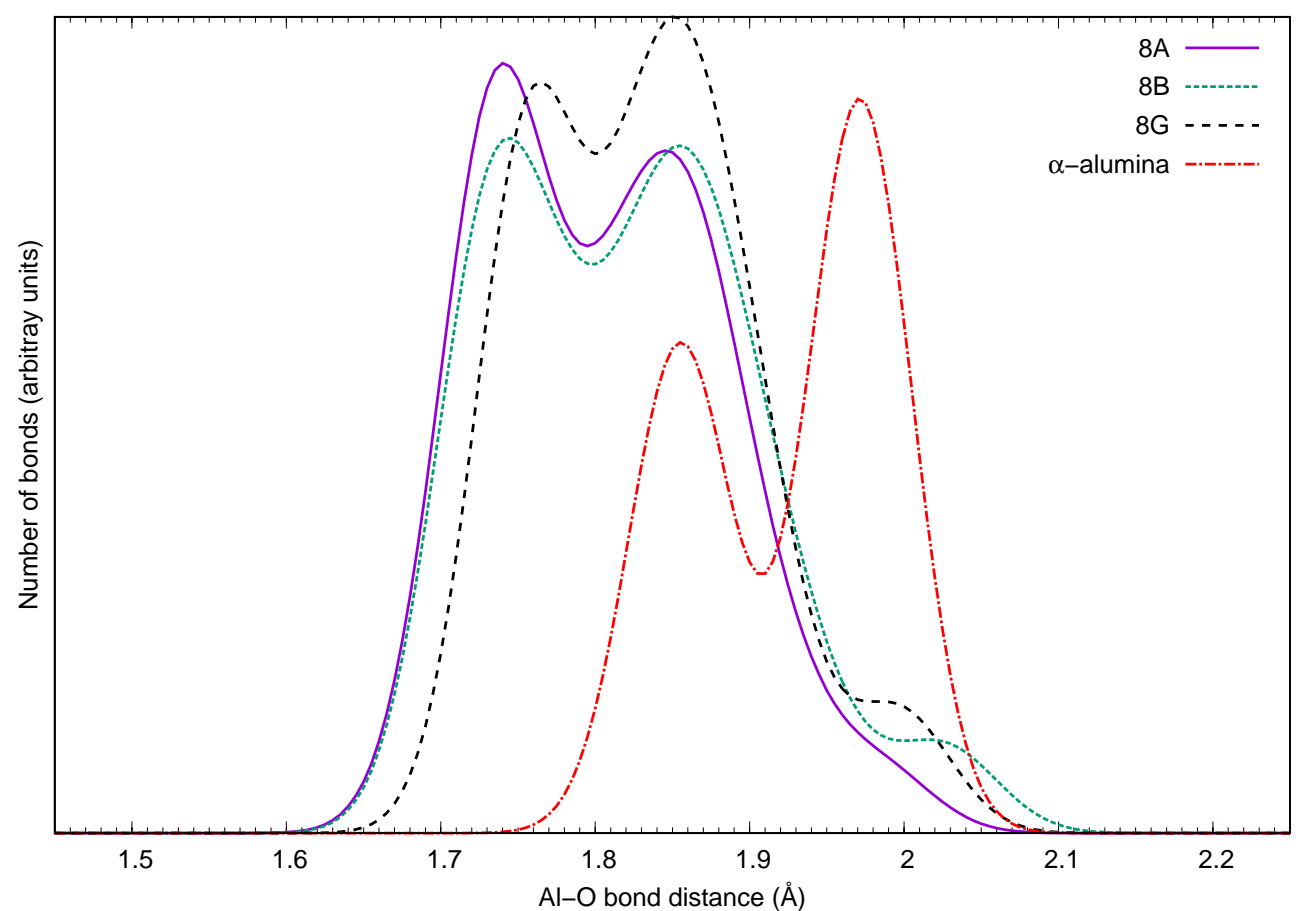

Figure 7: The Al-O bond lengths of $8 \mathrm{~A}, 8 \mathrm{~B}, 8 \mathrm{G}$ and $\alpha$-alumina. The curves are fitted with a Gaussian distribution and a half width $\gamma=0.03 \AA$.

Table 3: Mulliken charge analysis for isomers 8A, 8B, 8G and $\alpha$-alumina (in atomic units)

\begin{tabular}{l|cccc|c} 
charge & $8 \mathrm{~A}$ & $8 \mathrm{~B}$ & $8 \mathrm{G}$ & $8 \mathrm{I}$ & $\alpha$-alumina \\
\hline $\mathrm{q}(\overline{A l})$ & $0.42(0.30)$ & $0.34(0.24)$ & $0.22(0.09)$ & $0.46(0.36)$ & $1.38[30]$ \\
$\mathrm{q}(\bar{O})$ & $-0.28(-0.20)$ & $-0.23-(0.16)$ & $-0.15(-0.06)$ & $-0.31(-0.23)$ & $-0.92(=2 / 3 \times 1.38)$ \\
\hline
\end{tabular}

on the surface. Consequently, part of their atomic "neighbors" are missing and their coordination is lower.

In Table 3, the Mulliken charges of the presented clusters are shown. We also include a value for the average charge of $\alpha$-alumina in the bulk phase $(\mathrm{q}(\overline{A l})=+1.38 \mathrm{e},[30])$. However, we note that formal charge calculations strongly depend on the used basis set and functional and a comparison to the clusters is biased. In general, we find that the most stable isomers have higher formal atomic charges than the energetically less favourable clusters. Cluster 8I represents an exception, as it exhibits the largest average charges of all investigated clusters, but represents one of the energetically least stable ones due to its comparatively low coordination.

\subsection{Bond angles}

In Figure 8 , the Al-O-Al and $\mathrm{O}-\mathrm{Al}-\mathrm{O}$ bond angles are displayed for structures $8 \mathrm{~A}, 8 \mathrm{~B}$, $8 \mathrm{G}$ and $\alpha$-alumina. The latter exhibits characteristic angles at $79.6^{\circ}, 84.6^{\circ}, 86.4^{\circ}, 90.8^{\circ}$, 
$93.6^{\circ}$ and $132.2^{\circ}$ and $164.2^{\circ}$ degrees. The isomers $8 \mathrm{~A}$ and $8 \mathrm{~B}$ show a broad distribution of bond angles in the range between 80 and 180 degrees. Compared with $8 \mathrm{G}$, and the crystalline phase ( $\alpha$-alumina) the angle distribution of $8 \mathrm{~A}$ and $8 \mathrm{~B}$ is broader and has less pronounced peaks. The latter can be explained by the higher degree of symmetry in structure $8 \mathrm{G}$, since owing to its internal plane symmetry, every angle appears twice. The crystalline form of alumina shows the fewest and most pronounced peaks, owing to symmetry reasons.

The difference of the bulk to the clusters can be explained by the finite-size geometries of the clusters. Whereas in $\alpha$-alumina the periodicity of the crystal implies a homogeneous spatial occupation by the $\mathrm{Al}$ and $\mathrm{O}$ atoms, the clusters are largely empty in their interiors and the atoms reside on the surface. As a consequence the cluster bond angles are systematically larger than in $\alpha$-alumina.

\subsection{Vibrational analysis}

An alumina octomer has 40 atoms, consequently the number of vibrational degrees of freedom (i.e. vibration modes) is $3 \times 40-6=114$. The vibrational infrared (IR) spectra of $8 \mathrm{~A}, 8 \mathrm{~B}$, and $8 \mathrm{G}$ are shown as a function of wavelength in Figure 9. We use a Lorentzian function to describe the distribution of the peaks with a half-width at half-maximum $\gamma=$ 0.2 .

The majority of the IR modes of all shown clusters are located in a wavelength range between 10 and $20 \mu \mathrm{m}$ with a culmination point at 10-11 $\mu \mathrm{m}$. For structure $8 \mathrm{~A}$, the most intense vibration modes are located at wavelengths 10.012 (9.894), 10.985 (10.828), 10.729 (10.587) and 11.064 (10.921) $\mu \mathrm{m}$ for the B3LYP (PBE0) functional, respectively. For cluster 8B, the 10.348 (10.253), 10.126 (10.005), 11.009 (10.871) and $11.473(11.329) \mu \mathrm{m}$ for the B3LYP (PBE0) functional, respectively. These latter dominating vibrations are attributed to $\mathrm{Al}-\mathrm{O}$ stretching and bending modes. A complete table with all vibrational frequencies for $8 \mathrm{~A}$ and $8 \mathrm{~B}$ can be found in the Appendix.

We investigate isomers $8 \mathrm{~A}, 8 \mathrm{~B}$, and $8 \mathrm{G}$ also with the SDD (Stuttgart/Dresden) basis set including a vibrational analysis in order to benchmark with the results of ref. [19] who predicted isomer $8 \mathrm{G}$ as the global minimum. However, we found that vibrational calculations employing the SDD basis set led to imaginary frequencies for $8 \mathrm{~A}, 8 \mathrm{~B}, 8 \mathrm{G}$ which do not appear using a larger basis set $(6-311+G(p))$. We conclude that the SDD basis set is not adequate to describe alumina clusters and that all vibrational frequencies are real. Hence, clusters $8 \mathrm{~A}, 8 \mathrm{~B}$, and $8 \mathrm{G}$ correspond to real minimum structures (and not transition states).

The differences in the IR spectra between PBE0- and B3LYP-based calculations are small and the tiny relative shifts in wavelengths arise - among other reasons - due to the slightly shorter bond distances obtained with the PBE0 functional, compared to the B3LYP functional (as for the rotational constants).

The vibrational zero-point energies of isomers $8 \mathrm{~A}, 8 \mathrm{~B}$, and $8 \mathrm{G}$ are given in Table 4 They are of the order $3.5 \mathrm{eV}$ and vary by only $0.02 \mathrm{eV}$ among the cluster isomers $8 \mathrm{~A}$, $8 \mathrm{~B}$, and $8 \mathrm{G}$ within the same level of theory. Consequently, the relative energies of the clusters hardly shift and the energetic ordering is preserved by including the vibrational zero-point correction.

The vibration frequencies of the clusters $8 \mathrm{~A}, 8 \mathrm{~B}$, and $8 \mathrm{G}$ are shown in units of wavenumbers $\left(\mathrm{cm}^{-1}\right)$ in Table 7 in the Appendix. For clusters $8 \mathrm{~A}$ and $8 \mathrm{~B}$, the three 


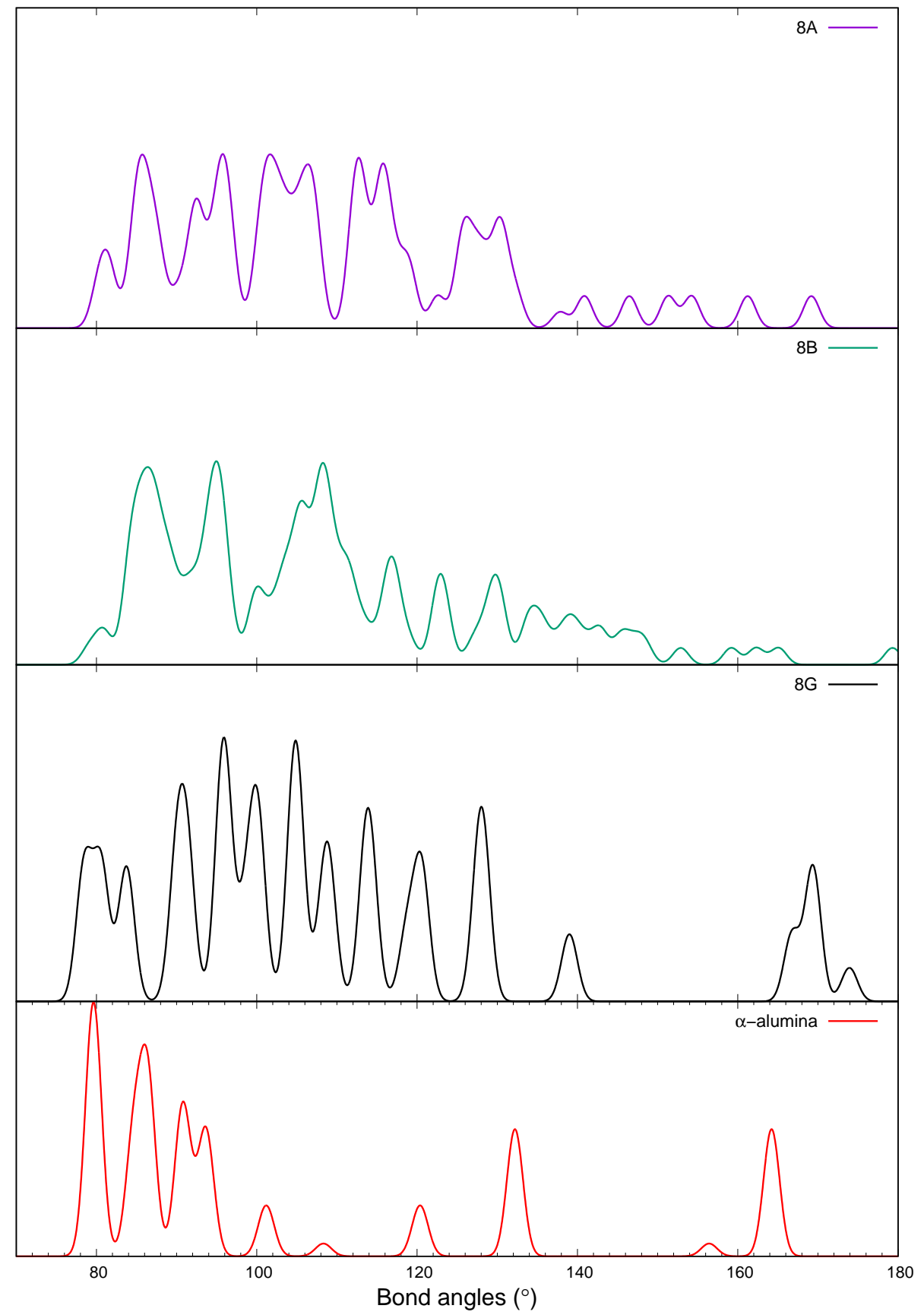

Figure 8: The Al-O-Al and O-Al-O bond angles of structures 8A 8B, 8G and alpha-alumina. The curves are fitted with a Gaussian distribution and a half width $\gamma=1.0^{\circ}$. 


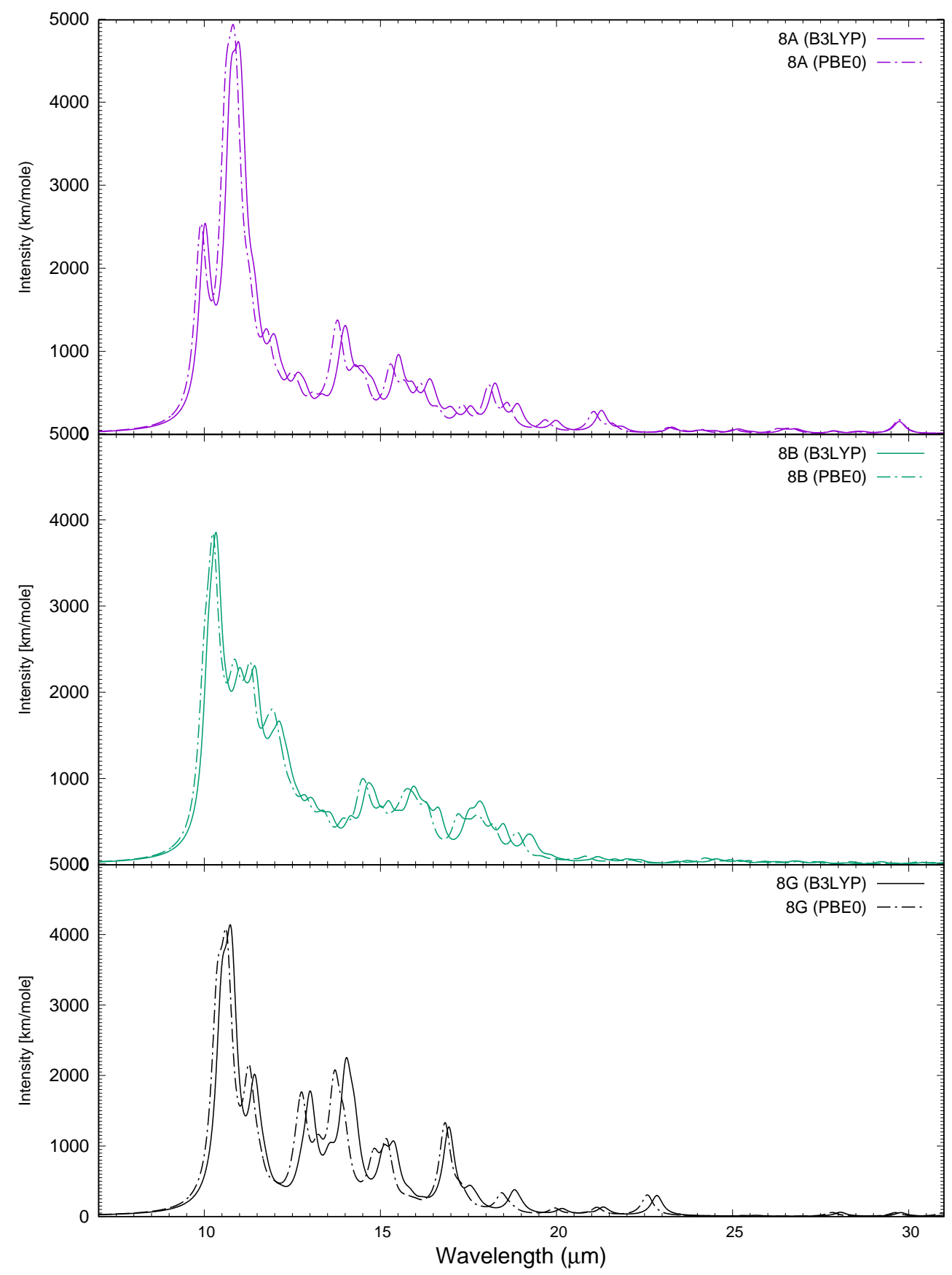

Figure 9: The vibration modes and related intensities of the newly-discovered clusters $8 \mathrm{~A}, 8 \mathrm{~B}$, and $8 \mathrm{G}$ fitted by a Lorentzian profile with a half width $\gamma=0.2$. Straight lines: B3LYP, dashed lines: PBE0. 
Table 4: Vibrational zero-point corrections $\epsilon_{0}($ in $\mathrm{eV}$ ) of $8 \mathrm{~A}, 8 \mathrm{~B}$, and $8 \mathrm{G}$ as calculated with the B3LYP and the PBE0 functional.

\begin{tabular}{l|rr} 
Isomer & $\epsilon_{0}(\mathrm{~B} 3 \mathrm{LYP})$ & $\epsilon_{0}(\mathrm{PBE} 0)$ \\
\hline $8 \mathrm{~A}$ & 3.50 & 3.54 \\
$8 \mathrm{~B}$ & 3.51 & 3.55 \\
$8 \mathrm{G}$ & 3.49 & 3.54
\end{tabular}

lowest modes are located slightly below $130 \mathrm{~cm}^{-1}$. In the harmonic oscillator approximation used in the present study, these low frequency modes are associated with hindered rotations rather than vibrations. No imaginary frequency occurs for structures $8 \mathrm{~A}, 8 \mathrm{~B}$ and $8 \mathrm{G}$.

\subsection{Thermochemistry}

We calculate thermochemical potentials (heat capacity $\mathrm{c}_{P}$, entropy $\mathrm{S}$, enthalpy of formation $\Delta \mathrm{H}_{f}(\mathrm{~T})$ and the (Gibbs) free energy of formation) $\Delta \mathrm{G}_{f}(\mathrm{~T})$ of both clusters $8 \mathrm{~A}$ and $8 \mathrm{~B}$ as a function of temperature (see Tables 5 and 6 in the Appendix). We use a script provided by 31 to calculate the heat capacity $c_{p}(\mathrm{~T})$, entropy $\mathrm{S}(\mathrm{T})$ and enthalpy $\mathrm{H}^{0}(\mathrm{~T})$ from the cluster calculation output. In order to determine $\mathrm{dH}_{f}$ and $\mathrm{dG}_{f}$, we follow the approach of Ochterski (see [28]) and make use of the enthalpies and the entropies of the elements $(\mathrm{Al}$ and $\mathrm{O})$ in their standard states listed the NIST-JANAF Thermochemical Tables ${ }^{1}$. As the fugacity of elemental gas-phase Al is 1 bar at 2790.81 $\mathrm{K}$ we constrain the temperature range to a maximum of $\mathrm{T}=2700 \mathrm{~K}$.

The thermodynamic tables for structures $8 \mathrm{~A}$ and $8 \mathrm{~B}$ can be found in the Appendix. They are evaluated at a pressure of $\mathrm{p}=1 \mathrm{~atm}$. At $0 \mathrm{~K}$ the entropy $\mathrm{dS}$ is vanishing by definition and $\mathrm{dH}_{f}$ equals $\mathrm{dG}_{f}$. We derive $\mathrm{dH}_{f}=-9114.5 \mathrm{~kJ} /$ mole for $8 \mathrm{~A}$ and $\mathrm{dH}_{f}=$ $-9100.3 \mathrm{~kJ} /$ mole for $8 \mathrm{~B}$ - in agreement with the relative potential energies including the zero-point correction. At $298.15 \mathrm{~K}$ the situation is similar and the relative enthalpies and Gibbs free energies differ by $13-17 \mathrm{~kJ} \mathrm{~mole}^{-1}$. Also for larger temperatures, structure $8 \mathrm{~A}$ is slightly more favored to form than structure $8 \mathrm{~B}$ as calculated within the B3IYP density functional. Around $\mathrm{T}=1400-1500 \mathrm{~K}$, the Gibbs free energy of formation for structures $8 \mathrm{~A}$ and $8 \mathrm{~B}$ changes sign and becomes positive (endergonic) for larger temperatures. The stability limits of the most stable, smaller-sized $(n<8)$ alumina clusters are similar to those of $8 \mathrm{~A}$ and $8 \mathrm{~B}$, and range from $\mathrm{T}=1300-1450 \mathrm{~K}$. We find a slight increase of the critical temperature with cluster size. Thus, an effective alumina octomer formation is expected to occur for temperatures below $1400 \mathrm{~K}$ and to be hampered for temperatures larger than $1500 \mathrm{~K}$ (at standard pressure of $1 \mathrm{~atm}$ ). Interestingly, the latter temperature coincidences with the condensation temperature for alumina grains in circumstellar conditions 32$]$.

\footnotetext{
${ }^{1}$ https://janaf.nist.gov/
} 


\section{Conclusion}

We report the discovery of $\operatorname{six}\left(\mathrm{Al}_{2} \mathrm{O}_{3}\right)_{8}$ isomers $(8 \mathrm{~A}, 8 \mathrm{~B}, 8 \mathrm{C}, 8 \mathrm{D}, 8 \mathrm{E}, 8 \mathrm{~F})$ that represent currently the lowest-energy alumina octomer structures known. The octomer is the smallest cluster size at which we find differences with previously reported candidate global minima. All six favourable octomer structures are neither hollow nor spherical, but exhibit elongated and "semi-compact" geometries. The IR spectra of the lowestenergy octomers is dominated by Al-O vibration modes in $10-11 \mu \mathrm{m}$ wavelength range. By comparing formal charges, bond lengths and angles of the reported low- energy octomers to those of crystalline $\alpha$-alumina, we conclude that, at a cluster size $n=8$, the alumina bulk limit is not (yet) reached. However, we find that the thermal stability limit of the octomers and smaller-sized polymers in the gas phase coincides with the glass-

liquid phase transition of liquid alumina.

Acknowledgements This work was supported by the ERC consolidator grant 646758 "AEROSOL". We acknowledge Dr. Amol D. Rahane for providing the cluster geometries from their studies for comparison. Moreover, we acknowledge the "Accordo Quadro INAF-CINECA (2017)", for the availability of high performance computing resources and support. This work was supported by a STSM Grant from COST Action CM1401 ("Our AstroChemical History"). This research was supported by the Spanish MINECO/FEDER CTQ2015-64618-R grant and, in part, by Generalitat de Catalunya (grants 2017SGR13 and XRQTC).

\section{References}

\section{References}

[1] R. P. Turco, R. C. Whitten, O. B. Toon, Stratospheric aerosols: Observation and theory, Reviews of Geophysics 20 (2) (1982) 233-279. arXiv:https://agupubs.onlinelibrary.wiley.com/doi/pdf/ 10.1029/RG020i002p00233 doi:10.1029/RG020i002p00233

URL https://agupubs . onlinelibrary.wiley.com/doi/abs/10.1029/RG020i002p00233

[2] Karovicova, I., Wittkowski, M., Ohnaka, K., Boboltz, D. A., Fossat, E., Scholz, M., New insights into the dust formation of oxygen-rich agb stars A\&A 560 (2013) A75. doi:10.1051/0004-6361/ 201322376

URL https://doi.org/10.1051/0004-6361/201322376

[3] Gobrecht, D., Cherchneff, I., Sarangi, A., Plane, J. M. C., Bromley, S. T., Dust formation in the oxygen-rich agb star ik tauri, A\&A 585 (2016) A6. doi:10.1051/0004-6361/201425363 URL https : //doi .org/10.1051/0004-6361/201425363

[4] Decin, L., Richards, A. M. S., Waters, L. B. F. M., Danilovich, T., Gobrecht, D., Khouri, T., Homan, W., Bakker, J. M., Van de Sande, M., Nuth, J. A., De Beck, E., Study of the aluminium content in agb winds using alma - indications for the presence of gas-phase (al2o3)n clusters, A\&A 608 (2017) A55. doi:10.1051/0004-6361/201730782

URL https://doi.org/10.1051/0004-6361/201730782

[5] A. Takigawa, T. Kamizuka, S. Tachibana, I. Yamamura, Dust formation and wind acceleration around the aluminum oxide-rich agb star w hydrae Science Advances 3 (11). arXiv:http:// advances.sciencemag.org/content/3/11/eaao2149.full.pdf, doi:10.1126/sciadv.aao2149 URL http://advances.sciencemag.org/content/3/11/eaao2149

[6] S. T. Bromley, J. C. Gomez Martin, J. M. C. Plane, Under what conditions does (sio)n nucleation occur? a bottom-up kinetic modelling evaluation. Phys. Chem. Chem. Phys. 18 (2016) 26913-26922. doi:10.1039/C6CP03629E URL http://dx.doi.org/10.1039/C6CP03629E

[7] H.-P. Gail, M. Scholz, A. Pucci, Silicate condensation in Mira variables, aap 591 (2016) A17. arXiv: 1604.04636 doi:10.1051/0004-6361/201628113 
[8] J. S. Bhatt, I. J. Ford, Investigation of mgo as a candidate for the primary nucleating dust species around $m$ stars, Monthly Notices of the Royal Astronomical Society 382 (1) (2007) 291298. arXiv:/oup/backfile/content_public/journal/mnras/382/1/10.1111/j.1365-2966.2007. 12358.x/2/mnras0382-0291.pdf |doi:10.1111/j.1365-2966.2007.12358.x

URL http://dx.doi.org/10.1111/j.1365-2966.2007.12358.x

[9] T. M. Koehler, H.-P. Gail, E. Sedlmayr, MgO dust nucleation in M-Stars: calculation of cluster properties and nucleation rates., aap 320 (1997) 553-567.

[10] L. R. Nittler, C. M. O. Alexander, R. Gallino, P. Hoppe, A. N. Nguyen, F. J. Stadermann, E. K. Zinner, Aluminum-, Calcium- and Titanium-rich Oxide Stardust in Ordinary Chondrite Meteorites, apj 682 (2008) 1450-1478. arXiv:0804.2866, doi:10.1086/589430

[11] F. Gyngard, E. Zinner, L. R. Nittler, A. Morgand, F. J. Stadermann, K. Mairin Hynes, Automated NanoSIMS Measurements of Spinel Stardust from the Murray Meteorite, apj 717 (2010) 107-120. arXiv: 1006.4355 , doi:10.1088/0004-637X/717/1/107

[12] L. D. Hart, Alumina chemicals Columbus, OH (USA); American Ceramic Society Inc., United States, 1990.

URL http://www.osti.gov/scitech/servlets/purl/5158074

[13] M. R. Noordin, K. Y. Liew, Synthesis of Alumina Nanofibers and Composites InTech, Rijeka, 2010. doi: $10.5772 / 8165$

URL http://dx.doi.org/10.5772/8165

[14] J.-H. Kim, S.-J. Yoo, D.-H. Kwak, H.-J. Jung, T.-Y. Kim, K.-H. Park, J.-W. Lee, Characterization and application of electrospun alumina nanofibers Nanoscale Research Letters 9 (1) (2014) 44. doi:10.1186/1556-276X-9-44 URL https://doi.org/10.1186/1556-276X-9-44

[15] V. Piriyawong, V. Thongpool, P. Asanithi, P. Limsuwan, Preparation and characterization of alumina nanoparticles in deionized water using laser ablation technique J. Nanomaterials 2012 (2012) $2: 2-2: 2$. doi: $10.1155 / 2012 / 819403$ URL http://dx.doi.org/10.1155/2012/819403

[16] I. P. Batra, Electronic structure of -al 2 o 3 Journal of Physics C: Solid State Physics 15 (26) (1982) 5399. URL http://stacks.iop.org/0022-3719/15/i=26/a=019

[17] D. van Heijnsbergen, K. Demyk, M. A. Duncan, G. Meijer, G. von Helden, Structure determination of gas phase aluminum oxide clusters, Phys. Chem. Chem. Phys. 5 (2003) 2515-2519. doi:10.1039/ B212654K

URL http://dx.doi.org/10.1039/B212654K

[18] M. Sierka, J. Dbler, J. Sauer, G. Santambrogio, M. Brmmer, L. Wste, E. Janssens, G. Meijer, K. Asmis, Unexpected structures of aluminum oxide clusters in the gas phase, Angewandte Chemie International Edition 46 (18) (2007) 3372-3375. arXiv:https://onlinelibrary.wiley.com/doi/ pdf/10.1002/anie.200604823 doi:10.1002/anie.200604823

URL https://onlinelibrary.wiley.com/doi/abs/10.1002/anie. 200604823

[19] A. B. Rahane, M. D. Deshpande, V. Kumar, Structural and electronic properties of (al2o3)n clusters with $\mathrm{n}=110$ from first principles calculations The Journal of Physical Chemistry C 115 (37) (2011) 18111-18121. arXiv:https://doi.org/10.1021/jp2050614 doi:10.1021/jp2050614 URL https://doi.org/10.1021/jp2050614

[20] R. Li, L. Cheng, Structural determination of $(\mathrm{al} 2 \mathrm{o} 3) \mathrm{n}(\mathrm{n}=17)$ clusters based on density functional calculation, Computational and Theoretical Chemistry 996 (2012) 125 - 131. doi:https://doi. org $/ 10.1016 / j$.comptc. 2012.07 .027

URL http://www.sciencedirect.com/science/article/pii/S2210271X12003726

[21] Q. Zhang, L. Cheng, Structural determination of $($ al2o3 $) \mathrm{n}(\mathrm{n}=115)$ clusters based on graphic processing unit, Journal of Chemical Information and Modeling 55 (5) (2015) 1012-1020, pMID: 25928795. arXiv:https://doi.org/10.1021/acs.jcim.5b00069 doi:10.1021/acs.jcim.5b00069. URL https://doi.org/10.1021/acs.jcim.5b00069

[22] D. Wales, J. Doye, Global Optimization by Basin-Hopping and the Lowest Energy Structures of Lennard-Jones Clusters Containing up to 110 Atoms, eprint arXiv:cond-mat/9803344 arXiv: cond-mat/9803344.

[23] S. T. Bromley, E. Flikkema, Columnar-to-disk structural transition in nanoscale $\left(\mathrm{sio}_{2}\right)_{N}$ clusters Phys. Rev. Lett. 95 (2005) 185505. doi:10.1103/PhysRevLett.95.185505 URL https://link.aps.org/doi/10.1103/PhysRevLett.95.185505

[24] S. M. Woodley, P. D. Battle, J. D. Gale, C. Richard A. Catlow, The prediction of inorganic crystal structures using a genetic algorithm and energy minimisation. Phys. Chem. Chem. Phys. 1 (1999) 
2535-2542. doi:10.1039/A901227C

URL http://dx.doi.org/10.1039/A901227C

[25] T. S. Bush, J. D. Gale, C. R. A. Catlow, P. D. Battle, Self-consistent interatomic potentials for the simulation of binary and ternary oxides, J. Mater. Chem. 4 (1994) 831-837. doi:10.1039/ JM9940400831

URL http://dx.doi.org/10.1039/JM9940400831

[26] A. D. Becke, A new mixing of Hartree-Fock and local density-functional theories, jcp 98 (1993) 1372-1377. doi:10.1063/1.464304

[27] J. P. Perdew, M. Ernzerhof, K. Burke, Rationale for mixing exact exchange with density functional approximations, jcp 105 (1996) 9982-9985. doi:10.1063/1.472933

[28] M. J. Frisch, G. W. Trucks, H. B. Schlegel, G. E. Scuseria, M. A. Robb, J. R. Cheeseman, G. Scalmani, V. Barone, B. Mennucci, G. A. Petersson, H. Nakatsuji, M. Caricato, X. Li, H. P. Hratchian, A. F. Izmaylov, J. Bloino, G. Zheng, J. L. Sonnenberg, M. Hada, M. Ehara, K. Toyota, R. Fukuda, J. Hasegawa, M. Ishida, T. Nakajima, Y. Honda, O. Kitao, H. Nakai, T. Vreven, J. A. Montgomery, Jr., J. E. Peralta, F. Ogliaro, M. Bearpark, J. J. Heyd, E. Brothers, K. N. Kudin, V. N. Staroverov, R. Kobayashi, J. Normand, K. Raghavachari, A. Rendell, J. C. Burant, S. S. Iyengar, J. Tomasi, M. Cossi, N. Rega, J. M. Millam, M. Klene, J. E. Knox, J. B. Cross, V. Bakken, C. Adamo, J. Jaramillo, R. Gomperts, R. E. Stratmann, O. Yazyev, A. J. Austin, R. Cammi, C. Pomelli, J. W. Ochterski, R. L. Martin, K. Morokuma, V. G. Zakrzewski, G. A. Voth, P. Salvador, J. J. Dannenberg, S. Dapprich, A. D. Daniels, . Farkas, J. B. Foresman, J. V. Ortiz, J. Cioslowski, D. J. Fox, Gaussian09 Revision E.01, gaussian Inc. Wallingford CT 2009

[29] Y. Gu, N. Xu, M. Lin, K. Tan, Structures, stabilities and properties of hollow (al2o3)n clusters $(\mathrm{n}=10,12,16,18,24$ and 33): Studied with density functional theory Computational and Theoretical Chemistry 1063 (2015) 29 -34. doi:https://doi.org/10.1016/j.comptc.2015.03.027 URL http://www.sciencedirect.com/science/article/pii/S2210271X15001425

[30] H. P. Pinto, R. M. Nieminen, S. D. Elliott, Ab initiostudy of $\gamma$-al2o3surfaces, Physical Review B 70 (12). doi:10.1103/physrevb.70.125402 URL https://doi.org/10.1103/physrevb.70.125402

[31] K. K. Irikura, D. J. Frurip, Computational Thermochemistry American Chemical Society, Washington, DC, 1998. arXiv:https://pubs.acs.org/doi/pdf/10.1021/bk-1998-0677, doi:10.1021/ bk-1998-0677 URL https://pubs.acs.org/doi/abs/10.1021/bk-1998-0677

[32] F. J. Molster, L. B. F. M. Waters, F. Kemper, The Mineralogy of Interstellar and Circumstellar Dust in Galaxies, in: T. Henning (Ed.), Lecture Notes in Physics, Berlin Springer Verlag, Vol. 815 of Lecture Notes in Physics, Berlin Springer Verlag, 2010, pp. 143-201. doi: 10.1007/978-3-642-13259-9_3

\section{Appendix}

Table 7: Vibration frequencies $\left(\right.$ in $\mathrm{cm}^{-1}$ ) of structures $8 \mathrm{~A}, 8 \mathrm{~B}$, and $8 \mathrm{G}$, respectively, as calculated on the B3LYP/6-311+G(d) level of theory.

$8 \mathrm{~A}$

$\begin{array}{rrrrrr}90.2094 & 103.6610 & 113.1299 & 113.7897 & 126.2836 & 132.1154 \\ 149.5395 & 156.1298 & 158.4286 & 161.9488 & 174.7097 & 178.0820 \\ 184.1340 & 190.3925 & 204.5866 & 208.6846 & 221.3737 & 224.7862 \\ 234.3400 & 239.6439 & 242.4419 & 247.5123 & 258.1091 & 263.3042 \\ 270.1901 & 274.4194 & 278.7248 & 281.9794 & 282.3983 & 287.7413 \\ 288.8951 & 296.8160 & 298.2015 & 304.0709 & 313.6256 & 317.7489 \\ 319.7042 & 323.7852 & 336.3023 & 341.3491 & 348.0401 & 349.9338 \\ 358.6037 & 368.5844 & 372.0300 & 373.5352 & 377.7690 & 385.1710 \\ 391.3964 & 396.4796 & 397.9887 & 408.3760 & 411.1994 & 415.4799 \\ 429.5667 & 445.8082 & 454.5025 & 457.4732 & 468.7610 & 470.0209\end{array}$




\begin{tabular}{|c|c|c|c|c|c|}
\hline 56 & 60 & 79 & & & \\
\hline 668 & 7.2123 & 569.4951 & 579.9749 & 588.3215 & 596.2581 \\
\hline & 111 & 9.6286 & .5216 & 8.7537 & 107 \\
\hline 739 & 6.3816 & 7.8524 & & & \\
\hline 378 & 0.3167 & 2.7577 & & & \\
\hline & & & & & \\
\hline & 9.7563 & & & & \\
\hline 32 & 7 & 19 & & & \\
\hline 530 & & & & & \\
\hline \multicolumn{6}{|l|}{$8 \mathrm{~B}$} \\
\hline 88 & 2 & 9 & & 8 & 255 \\
\hline 9458 & & & & & \\
\hline & 17 & & & & \\
\hline & & & & & \\
\hline & & & & & \\
\hline & & & & & \\
\hline & 25 & 36 & & & \\
\hline & & & & & \\
\hline & & & & & \\
\hline & & & & & \\
\hline & & & & & \\
\hline & & & & & \\
\hline & & & & & \\
\hline & & & & & \\
\hline & & & & & \\
\hline & & & & & \\
\hline & & & & & \\
\hline & & & & & \\
\hline 380 & & & & & \\
\hline \multicolumn{6}{|l|}{80} \\
\hline & & & & & \\
\hline & & & & & 287 \\
\hline & & & & & \\
\hline & & & & & \\
\hline & & & & & \\
\hline & & & & & 252 \\
\hline & & & & & 173 \\
\hline 699 & 38 & & & 7900 & 6817 \\
\hline & & & & & 270 \\
\hline & & & & & \\
\hline & & & & & \\
\hline & & & & & \\
\hline & & & & & \\
\hline & 634.1 & 53 & 13 & 643.1729 & 379 \\
\hline & & & & & $101+8$ \\
\hline & 120.0900 & 120.0001 & 131.1200 & 737.7345 & 757.050 \\
\hline
\end{tabular}


$\begin{array}{llllll}768.7273 & 768.7298 & 783.9297 & 788.5174 & 816.6344 & 822.2092\end{array}$

$\begin{array}{lllllll}822.2348 & 850.9110 & 853.7232 & 853.8241 & 857.5930 & 865.4159\end{array}$

$\begin{array}{llllll}874.7006 & 874.7399 & 898.7439 & 928.8732 & 928.9094 & 952.6200\end{array}$ 
Table 5: Thermodynamic quantities (temperature $\mathrm{T}$, entropy $\mathrm{S}$, heat capacity $\mathrm{c}_{P}$, enthalpy change $\mathrm{H}(\mathrm{T})-\mathrm{H}(0)$, enthalpy of formation $\mathrm{dH}_{f}$ and Gibbs free energy of formation $\mathrm{dG}_{f}$ of $8 \mathrm{~A}$. Units are $\mathrm{K}$ for $\mathrm{T}, \mathrm{JK}^{-1} \mathrm{~mol}^{-1}$ for $\mathrm{S}$ and $\mathrm{c}_{P}$, and $\mathrm{kJmol}^{-1}$ for $\mathrm{H}(\mathrm{T})-\mathrm{H}(0), \mathrm{dH}_{f}$ and $\mathrm{dG}_{f}$.

\begin{tabular}{l|rrrrr}
$\mathrm{T}$ & $\mathrm{S}$ & $\mathrm{C}_{P}$ & $\mathrm{H}(\mathrm{T})-\mathrm{H}(0)$ & $\mathrm{dH}_{f}(\mathrm{~kJ} / \mathrm{m})$ & $\mathrm{dG}_{f}$ \\
\hline 0.00 & 0.000 & 0.000 & 0.000 & -9114.469 & -9114.469 \\
100.00 & 395.149 & 205.289 & 9.052 & -9055.689 & -8545.540 \\
200.00 & 619.018 & 459.222 & 42.819 & -8992.442 & -7887.085 \\
250.00 & 732.484 & 558.040 & 68.335 & -8957.766 & -7554.811 \\
298.15 & 837.577 & 634.649 & 97.118 & -8922.095 & -7234.368 \\
300.00 & 841.510 & 637.258 & 98.295 & -8920.662 & -7222.025 \\
350.00 & 944.617 & 699.536 & 131.778 & -8881.499 & -6889.260 \\
400.00 & 1041.339 & 748.222 & 168.022 & -8840.751 & -6556.870 \\
450.00 & 1131.760 & 786.395 & 206.426 & -8798.843 & -6225.041 \\
500.00 & 1216.237 & 816.552 & 246.528 & -8756.149 & -5893.967 \\
600.00 & 1369.237 & 860.003 & 330.512 & -8669.469 & -5234.332 \\
700.00 & 1504.114 & 888.813 & 418.046 & -8582.487 & -4578.544 \\
800.00 & 1624.174 & 908.693 & 507.979 & -8496.474 & -3927.026 \\
900.00 & 1732.067 & 922.903 & 599.597 & -8412.296 & -3280.074 \\
1000.00 & 1829.874 & 933.372 & 692.436 & -8500.065 & -2807.347 \\
1100.00 & 1919.221 & 941.289 & 786.186 & -8415.723 & -2164.472 \\
1200.00 & 2001.398 & 947.410 & 880.634 & -8331.227 & -1523.173 \\
1300.00 & 2077.429 & 952.234 & 975.625 & -8246.644 & -883.305 \\
1400.00 & 2148.144 & 956.101 & 1071.049 & -8162.028 & -244.770 \\
1500.00 & 2214.219 & 959.246 & 1166.822 & -8077.399 & 392.473 \\
1600.00 & 2276.212 & 961.836 & 1262.880 & -7992.845 & 1028.525 \\
1700.00 & 2334.590 & 963.995 & 1359.174 & -7908.359 & 1663.434 \\
1800.00 & 2389.743 & 965.812 & 1455.667 & -7823.970 & 2297.288 \\
1900.00 & 2442.004 & 967.356 & 1552.328 & -7739.733 & 2930.067 \\
2000.00 & 2491.658 & 968.678 & 1649.131 & -7655.602 & 3561.866 \\
2100.00 & 2538.948 & 969.819 & 1746.057 & -7571.660 & 4192.683 \\
2200.00 & 2584.087 & 970.810 & 1843.090 & -7487.851 & 4822.595 \\
2300.00 & 2627.261 & 971.677 & 1940.215 & -7404.230 & 5451.636 \\
2400.00 & 2668.632 & 972.439 & 2037.422 & -7320.775 & 6079.827 \\
2500.00 & 2708.343 & 973.113 & 2134.700 & -7237.505 & 6707.158 \\
2600.00 & 2746.521 & 973.711 & 2232.042 & -7154.387 & 7333.788 \\
2700.00 & 2783.279 & 974.245 & 2329.440 & -7071.437 & 7959.704 \\
& & & & &
\end{tabular}


Table 6: Thermodynamic quantities (temperature $\mathrm{T}$, entropy $\mathrm{S}$, heat capacity $\mathrm{c}_{P}$, enthalpy change $\mathrm{H}(\mathrm{T})-\mathrm{H}(0)$, enthalpy of formation $\mathrm{dH}_{f}$ and Gibbs free energy of formation $\mathrm{dG}_{f}$ of isomer $8 \mathrm{~B}$. Units are $\mathrm{K}$ for $\mathrm{T}, \mathrm{JK}^{-1} \mathrm{~mol}^{-1}$ for $\mathrm{S}$ and $\mathrm{c}_{P}$, and $\mathrm{kJmol}^{-1}$ for $\mathrm{H}(\mathrm{T})-\mathrm{H}(0), \mathrm{dH}_{f}$ and $\mathrm{dG}_{f}$.

\begin{tabular}{l|rrrrr}
$\mathrm{T}$ & & $\mathrm{C}_{P}$ & $\mathrm{H}(\mathrm{T})-\mathrm{H}(0)$ & $\mathrm{dH}_{f}$ & $\mathrm{dG}_{f}$ \\
\hline 0.00 & 0.000 & 0.000 & 0.000 & -9100.292 & -9100.292 \\
100.00 & 387.576 & 197.744 & 8.595 & -9041.969 & -8531.063 \\
200.00 & 607.633 & 456.565 & 41.841 & -8979.243 & -7871.609 \\
250.00 & 720.715 & 557.171 & 67.273 & -8944.651 & -7538.753 \\
298.15 & 825.748 & 634.774 & 96.041 & -8908.995 & -7217.741 \\
300.00 & 829.682 & 637.411 & 97.217 & -8907.563 & -7205.378 \\
350.00 & 932.857 & 700.208 & 130.723 & -8868.377 & -6872.022 \\
400.00 & 1029.685 & 749.124 & 167.007 & -8827.589 & -6539.047 \\
450.00 & 1120.218 & 787.372 & 205.458 & -8785.634 & -6206.638 \\
500.00 & 1204.798 & 817.523 & 245.610 & -8742.890 & -5874.989 \\
600.00 & 1357.967 & 860.870 & 329.685 & -8656.119 & -5214.220 \\
700.00 & 1492.968 & 889.549 & 417.300 & -8569.056 & -4557.311 \\
800.00 & 1613.118 & 909.310 & 507.301 & -8482.975 & -3904.682 \\
900.00 & 1721.078 & 923.422 & 598.975 & -8398.741 & -3256.628 \\
1000.00 & 1818.935 & 933.812 & 691.862 & -8486.462 & -2782.805 \\
1100.00 & 1908.322 & 941.664 & 785.653 & -8402.079 & -2138.839 \\
1200.00 & 1990.528 & 947.733 & 880.135 & -8317.549 & -1496.451 \\
1300.00 & 2066.583 & 952.515 & 975.157 & -8232.935 & -855.496 \\
1400.00 & 2137.318 & 956.347 & 1070.607 & -8148.293 & -215.879 \\
1500.00 & 2203.409 & 959.462 & 1166.402 & -8063.642 & 422.445 \\
1600.00 & 2265.415 & 962.028 & 1262.481 & -7979.067 & 1059.578 \\
1700.00 & 2323.804 & 964.166 & 1358.794 & -7894.562 & 1695.567 \\
1800.00 & 2378.967 & 965.966 & 1455.303 & -7810.157 & 2330.498 \\
1900.00 & 2431.236 & 967.495 & 1551.978 & -7725.906 & 2964.353 \\
2000.00 & 2480.896 & 968.804 & 1648.795 & -7641.761 & 3597.231 \\
2100.00 & 2528.192 & 969.934 & 1745.733 & -7557.807 & 4229.124 \\
2200.00 & 2573.337 & 970.915 & 1842.776 & -7473.988 & 4860.108 \\
2300.00 & 2616.515 & 971.774 & 1939.912 & -7390.356 & 5490.226 \\
2400.00 & 2657.889 & 972.528 & 2037.128 & -7306.892 & 6119.493 \\
2500.00 & 2697.604 & 973.195 & 2134.415 & -7223.613 & 6747.897 \\
2600.00 & 2735.785 & 973.787 & 2231.764 & -7140.488 & 7375.601 \\
2700.00 & 2772.546 & 974.315 & 2329.170 & -7057.530 & 8002.590 \\
& & & & &
\end{tabular}

